\title{
Direct Experimental Characterization of the Ferrier Glycosyl Cation in the Gas Phase
}

Kim Greis, ${ }^{[\mathrm{a}],[\mathrm{b}]}$ Carla Kirschbaum, ${ }^{[\mathrm{a}],[\mathrm{b}]}$ Sabrina Leichnitz, ${ }^{[\mathrm{a}],[\mathrm{c}]}$ Sandy Gewinner, ${ }^{[\mathrm{b}]}$ Wieland

Schöllkopf, ${ }^{[b]}$ Gert von Helden, ${ }^{[b]}$ Gerard Meijer, ${ }^{[b]}$ Peter H. Seeberger, ${ }^{[a],[c]}$ Kevin Pagel ${ }^{*[a],[b]}$

[a] Freie Universität Berlin, Institute of Chemistry and Biochemistry, Berlin, Germany

[b] Fritz Haber Institute of the Max Planck Society, Berlin, Germany

[c] Max Planck Institute of Colloids and Interfaces, Potsdam, Germany

Supporting Information Placeholder

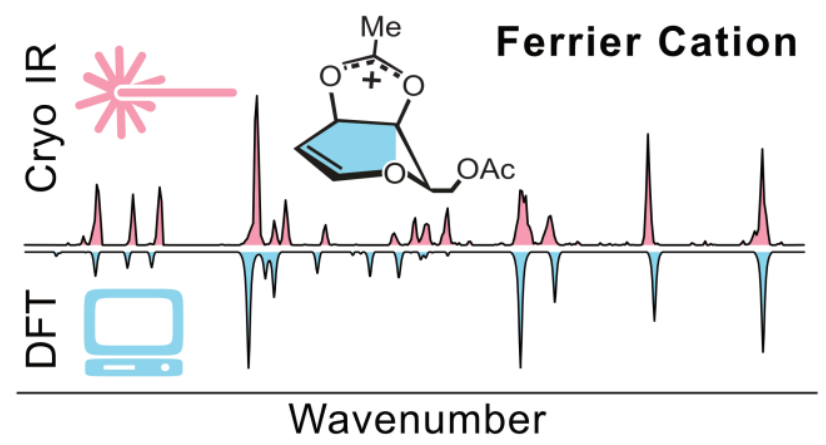

For Table of Contents Only

\begin{abstract}
The Ferrier rearrangement reaction is crucial for the synthesis of pharmaceuticals. Although its mechanism was described more than 50 years ago, the structure of the intermediate remains elusive. Two structures have been proposed for this Ferrier glycosyl cation: a 1,2-unsaturated cation that is resonance stabilized within the pyranose ring or a cation that is stabilized by anchimeric assistance of a neighboring acetyl group. Using a combination of gas-phase cryogenic infrared spectroscopy in helium nanodroplets and first-principles density-functional theory, we provide the first direct structural characterization of Ferrier cations. The data shows that both acetylated glucal and galactal lead to glycosyl cations of the dioxolenium-type.
\end{abstract}

In 1917, Emil Fischer reported a class of 1,2-unsaturated monosaccharide building blocks, so-called glycals. ${ }^{1}$ Almost 50 years later, the rich chemistry of glycals was exploited by Robert J. Ferrier, who first accurately described the mechanism of the allylic rearrangement in protected glycals upon nucleophilic substitution, today known as the Ferrier rearrangement (Scheme 1). ${ }^{2-3}$ In this reaction, the C3-acetyl group in fully acetylated glycals is cleaved to form the "Ferrier" glycosyl cation that in turn is attacked by nucleophiles at the $\mathrm{C} 1$-position. After an allylic shift, the reaction yields 2,3-unsaturated glycosides - a class of glycosides that is relevant for the synthesis of natural products ${ }^{4}$ and pharmaceuticals such as 2-deoxyglycosides, ${ }^{5} \mathrm{C}$-glycopyranosyl arenes and heteroarenes. ${ }^{6}$ The reaction can also be observed for glycals bearing other participating protecting groups, i.e. benzoyl. ${ }^{3}$

Scheme 1. Ferrier rearrangement reaction: activation of acetylated glucal leads to Ferrier glucosyl cations that can be attacked by nucleophiles.

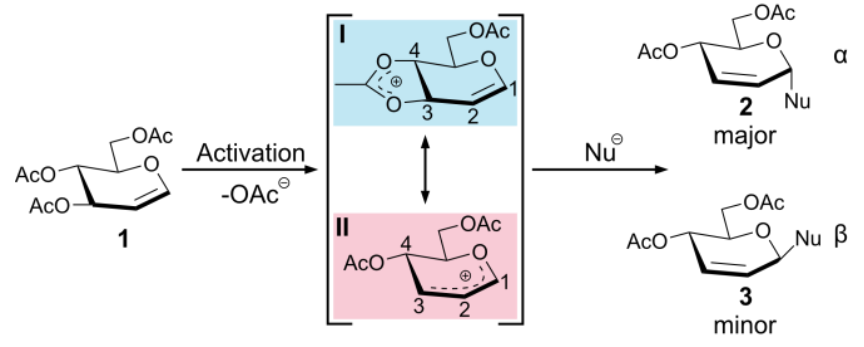


Due to their short-lived nature, the exact structure of the intermediate of the Ferrier rearrangement remains elusive. It has been postulated that cleavage of the $\mathrm{C} 3$-acetyl group is eased by neighboring group participation (anchimeric assistance) of the C4acetyl group, leading to a dioxolenium-type ion $\mathbf{I} .{ }^{7}$ However, so far no direct experimental evidence has been available to determine whether the underlying structural motif is indeed a dioxolenium ion I or a resonance stabilized oxocarbenium ion II. Recently, a Ferrier cation with protonated acetyl groups was characterized by NMR spectroscopy in superacids. As protonation of acetyl groups drastically reduces nucleophilicity, participation cannot be observed under these conditions. ${ }^{8}$ In a previous study on glycosyl cations, it was shown that $\mathrm{C} 4$-acetyl groups, contrary to $\mathrm{C} 2$-acetyl groups, do not participate in superacids. ${ }^{9}$

In the last years, it has been demonstrated that glycosyl cations can be isolated in the gas phase and characterized using infrared (IR) spectroscopy. In combination with density-functional theory (DFT), the underlying structural motif can be unambiguously identified. Thereby, it has been shown that neighboring and remote acetyl groups can stabilize a glycosyl cation by forming a covalent bond to the anomeric carbon. ${ }^{10-14}$ Here, we use gas-phase cryogenic IR spectroscopy and DFT to elucidate the structure of the Ferrier glycosyl cations generated from acetylated glucal and galactal.

A detailed description of the experimental setup can be found in the SI. Briefly, Ferrier cations are generated by nanoelectrospray ionization (nESI) followed by in-source fragmentation of sodiated glycal precursor ions. Representative mass spectra (Figure 1) reveal that fragmentation readily occurs for acetylated glycals, while it cannot be observed for unprotected glycals (Figures S1S2). This is mainly attributed to acetate being a better leaving group than hydroxide and the anchimeric assistance of neighboring acetyl groups.

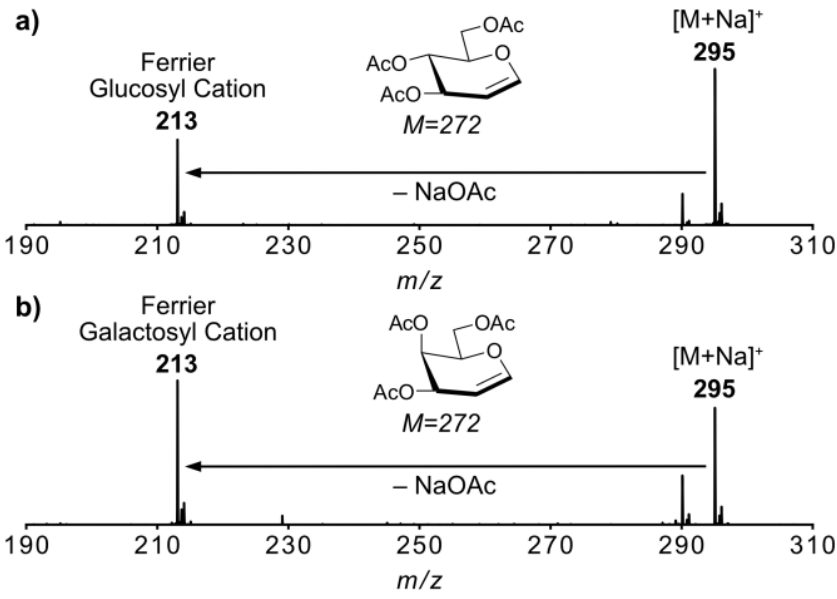

Figure 1. ESI-(+)-mass spectra of (a) 3,4,6-tri- $O$-acetyl-D-glucal and (b) 3,4,6-tri- $O$-acetyl-D-galactal, detected as $[\mathrm{M}+\mathrm{Na}]^{+}$at $\mathrm{m} / \mathrm{z}$ 295. The $[\mathrm{M}+\mathrm{Na}]^{+}$ions undergo loss of $\mathrm{NaOAc}$ under in-source CID conditions leading to Ferrier cations at $m / z$ 213. [M+NH$\left.]_{4}\right]^{+}$ adducts can be observed at $\mathrm{m} / \mathrm{z} 290$.

The generated Ferrier cations are isolated using a quadrupole mass filter and subsequently accumulated in a hexapole ion trap, where the ions are cooled to $90 \mathrm{~K}$. A beam of superfluid helium nanodroplets is guided through the ion trap, picking up analyte ions, cooling them to $0.4 \mathrm{~K}$ and leading them to a detection region. Here, the beam of ion-doped helium droplets overlaps with an IR beam $\left(1000-1800 \mathrm{~cm}^{-1}\right)$ that is produced by the Fritz Haber Institute free-electron laser (FHI FEL) ${ }^{15}$ The absorption of multiple resonant IR photons leads to release of the analyte ions from the droplets that are subsequently detected by a time-of-flight mass spectrometer. Plotting the ion count as a function of the IR wavenumber leads to a highly-resolved IR spectrum of the analyzed ion.

To derive structural information, the experimental IR spectra were linked to quantum chemically modelled structures. The conformational space of the Ferrier cations was explored using the genetic algorithm FAFOOM that allows for sampling rotatable bonds and ring puckers. ${ }^{16}$ Each generated structure was pre-optimized by the dispersion corrected $\mathrm{PBE}+\mathrm{vdW} \mathrm{W}^{\mathrm{TS}}$ density functional as implemented in FHI-aims using light basis set settings. ${ }^{17-18}$ The conformational search mainly yielded dioxolenium ions I and oxocarbenium ions II, but also dioxolenium-type structures in which the C4-acetyl group interacts remotely with the anomeric carbon (remote participation). A subset of the lowest-energy structures of each type of ions was reoptimized and frequencies computed at PBE0+D3/6-311+G(d,p) level of theory using Gaussian 16. ${ }^{19-21}$ The computed IR spectra were normalized and scaled by a factor of 0.965 . Cremer-Pople coordinates were used to assign ring puckers. ${ }^{22-23}$ Energetics, ring puckers and coordinates of the reoptimized structures can be found in the SI.

The IR spectrum of the Ferrier glucosyl cation shows a complex signature that can be interpreted using the computed spectra of low-energy conformers (Figure 2a). The experimental IR signature matches the theoretical spectrum of the dioxolenium-type glycosyl cation $\mathbf{I}$, exhibiting a ${ }^{5} \boldsymbol{E}$ ring pucker in which the carbonyl oxygen of the C4-acetyl group forms a covalent bond (1.53 $\AA$ ) with the C3-atom (Figure 2b). The free energy of this structural motif is generally more favorable than that of oxocarbenium ions II that adopt a distorted ${ }^{5} \boldsymbol{E}$ ring pucker without direct participation of protecting groups (Figure 2c). Generally, the most diagnostic absorption bands are found above $1500 \mathrm{~cm}^{-1}$. Bands at 1506 and $1550 \mathrm{~cm}^{-1}$ correspond to the symmetric and antisymmetric C-O-C stretches, while the signal at $1646 \mathrm{~cm}^{-1}$ represents a $\mathrm{C}=\mathrm{C}$ stretching vibration. The pronounced absorption band at $1750 \mathrm{~cm}^{-1}$ can be attributed to the $\mathrm{C}=\mathrm{O}$ stretch. Features below $1300 \mathrm{~cm}^{-1}$ result from $\mathrm{C}-\mathrm{C}$ and $\mathrm{C}-\mathrm{O}$ stretching vibrations, while the bands around $1400 \mathrm{~cm}^{-1}$ are due to $\mathrm{C}-\mathrm{H}$ bending modes. Although the absorption bands in the region below $1500 \mathrm{~cm}^{-1}$ are usually less diagnostic, theory fits the experiment surprisingly well. The spectral signature of the energetically unfavored $\left(+29 \mathrm{~kJ} \mathrm{~mol}^{-1}\right)$ oxocarbenium-type species II on the other hand does not match the experimental spectrum. While some signals seem to overlap, others are not present in the experimental spectrum and vice versa. In addition, none of the oxocarbenium-type species II matches the experimental spectrum particularly well. The spectra of the energetically less favored dioxolenium ion featuring remote participation $\left(+49 \mathrm{~kJ} \mathrm{~mol}^{-1}\right)$ do also not correspond to the experimental spectrum (Figure S7). It can therefore be concluded that the underlying structural motif of the Ferrier glucosyl cation exhibits anchimeric assistance of the C4acetyl group to form dioxolenium-type I structures. 
a)

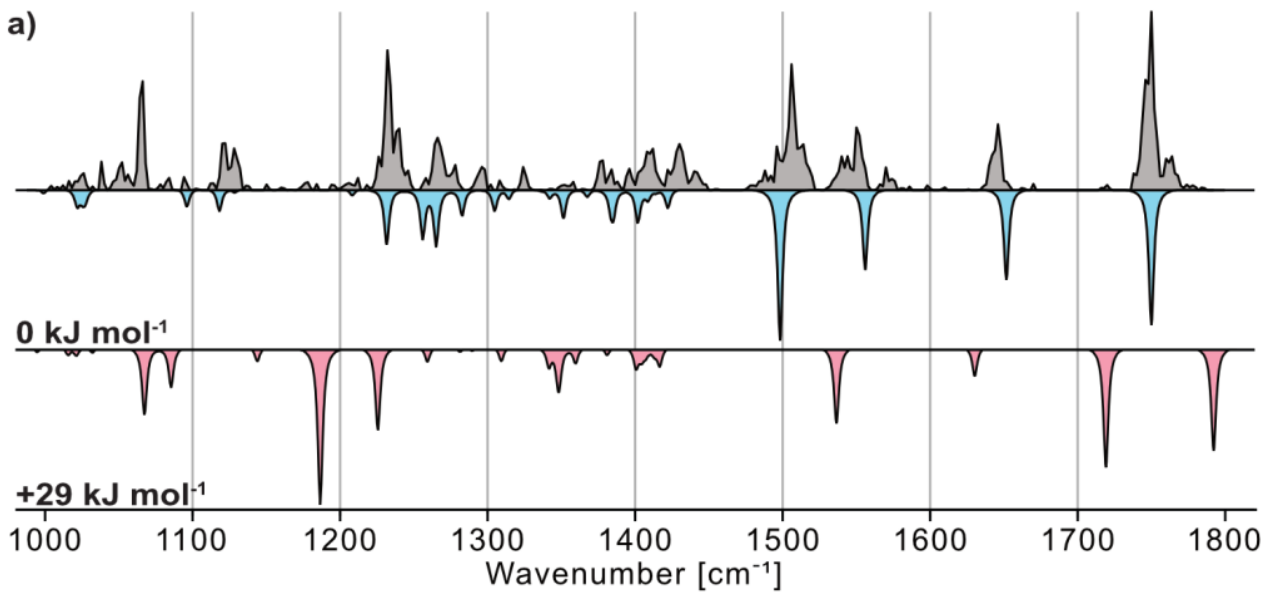

b)

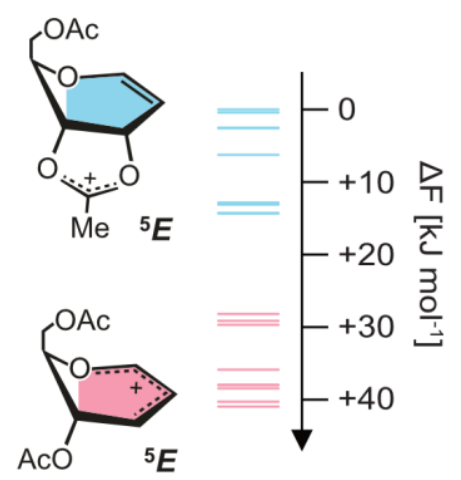

Figure 2. (a) Infrared spectra of Ferrier glucosyl cations generated from fully acetylated glucal precursor. The experimental IR spectrum is shown as grey trace, while computed spectra of low-energy dioxolenium (blue) and oxocarbenium (red) structures are represented in the inverted traces below. (b) Simplified representation of the low-energy structures and puckers. (c) Free energies of reoptimized low-energy structures at $90 \mathrm{~K}$.

a)

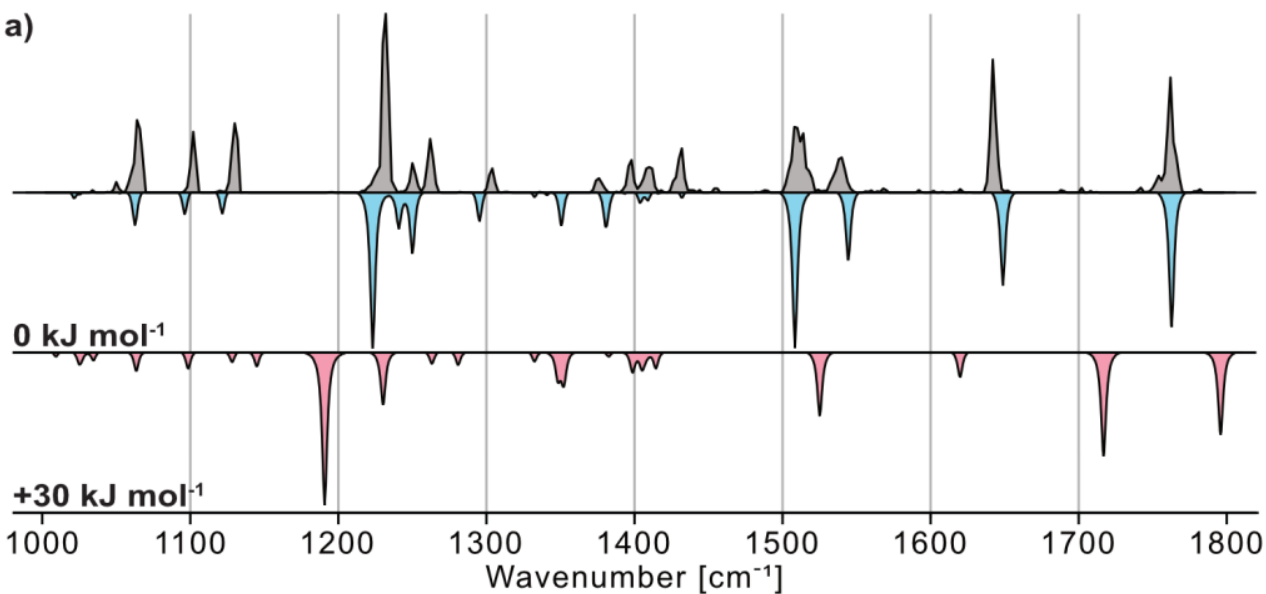

b)

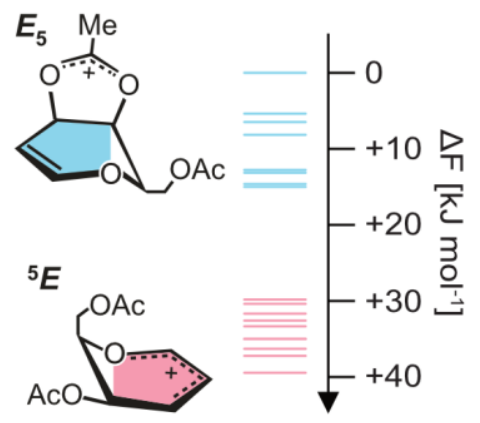

Figure 3. (a) Infrared spectra of Ferrier galactosyl cations generated from fully acetylated galactal precursor. The experimental IR spectrum is shown as grey trace, while computed spectra of low-energy dioxolenium (blue) and oxocarbenium (red) structures are represented in the inverted traces below. (b) Simplified representation of the low-energy structures and puckers. (c) Free energies of reoptimized low-energy structures at $90 \mathrm{~K}$.

Similar results were obtained for the Ferrier galactosyl cation (Figure 3a). The computed spectrum of dioxolenium-type $\mathbf{I}$ structure overwhelmingly matches the experimental spectrum. Besides the highly diagnostic vibrations above $1500 \mathrm{~cm}^{-1}$, here also the fingerprint region between 1000 to $1300 \mathrm{~cm}^{-1}$ agrees exceptionally well. Again, the dioxolenium-type $\mathbf{I}$ ion features a covalent bond $(1.52 \AA)$ between the carbonyl oxygen of the $\mathrm{C} 4$-acetyl group and the $\mathrm{C} 3$-atom and adopts an $\boldsymbol{E}_{5}$ ring pucker (Figure $3 \mathrm{~b}$ ). The lowest-energy oxocarbenium-type II structure adopts a ${ }^{5} \boldsymbol{E}$ ring pucker, but exhibits a free energy that is $30 \mathrm{~kJ} \mathrm{~mol}^{-1}$ larger than that of the lowest-energy dioxolenium-type I structure (Figure 3c). Not surprisingly, the spectral signatures of the oxocarbenium-type II structures generally match the experiment rather poorly. The Ferrier galactosyl cation adopts a dioxolenium-type I structure, formed by neighboring group participation of the C4-acetyl group.

In summary, we unraveled the detailed molecular structure of the key intermediate of the Ferrier rearrangement reaction. Direct experimental characterization by gas-phase cryogenic IR spectroscopy combined with first-principles density-functional theory unambiguously revealed that dioxolenium-type ions are the underlying structural motif of Ferrier glycosyl cations. In cations generated from both glucal and galactal, stabilization of the positive charge at $\mathrm{C} 3$ via participation of the C4-acetyl group is clearly favored over delocalization of the charge within the pyranose ring. The findings provide unprecedented insights into the mechanism of the Ferrier rearrangement and showcase, how such experiments can support the development of novel building blocks for the synthesis of 2,3-unsaturated glycosides.

\section{ASSOCIATED CONTENT}

\section{Supporting Information}

A detailed description of the experiment, mass spectra, computed energetics, spectra and structures are included in the supporting information. 
The Supporting Information is available free of charge on the ACS Publications website.

\section{AUTHOR INFORMATION}

\section{Corresponding Author}

Prof. Dr. Kevin Pagel

E-Mail: kevin.pagel@fu-berlin.de

orcid.org/0000-0001-8054-4718

\author{
Authors \\ Kim Greis \\ orcid.org/0000-0002-9107-2282 \\ Carla Kirschbaum \\ orcid.org/0000-0003-3192-0785 \\ Sabrina Leichnitz

\section{Sandy Gewinner} \\ Dr. Wieland Schöllkopf \\ orcid.org/0000-0003-0564-203X \\ Prof. Dr. Gert von Helden \\ orcid.org/0000-0001-7611-8740 \\ Prof. Dr. Gerard Meijer \\ orcid.org/0000-0001-9669-8340 \\ Prof. Dr. Peter H. Seeberger \\ orcid.org/0000-0003-3394-8466
}

\section{Notes}

The authors declare no competing financial interests.

\section{ACKNOWLEDGMENT}

The authors acknowledge generous funding by the European Research Council, ERC-2019-CoG-863934-GlycoSpec. KG is grateful to the Fonds National de la Recherche (FNR), Luxembourg, for funding the project GlycoCat (13549747). CK is grateful for financial support by Fonds der Chemischen Industrie.

\section{REFERENCES}

1. Fischer, E., Über neue Reduktionsprodukte des Traubenzuckers: Glucal und Hydro-glucal. Ber. Dtsch. Chem. Ges. 1917, 47, 196.

2. Ferrier, R. J.; Overend, W. G.; Ryan, A. E., 712. The reaction between 3,4,6-tri-O-acetyl-D-glucal and p-nitrophenol. $J$. Chem. Soc. 1962.

3. Ferrier, R. J.; Zubkov, O. A., Transformation of Glycals into 2,3-Unsaturated Glycosyl Derivatives. Org. React. 2003, 62.

4. Fraser-Reid, B.; Lopez, J., Unsaturated Sugars: A Rich Platform for Methodological and Synthetic Studies. Curr. Org. Chem. 2009, 13 (6), 532-553.

5. Bennett, C. S.; Galan, M. C., Methods for 2-Deoxyglycoside Synthesis. Chem. Rev. 2018, 118 (17), 7931-7985.

6. Bokor, E.; Kun, S.; Goyard, D.; Toth, M.; Praly, J. P.; Vidal, S.; Somsak, L., C-Glycopyranosyl Arenes and Hetarenes: Synthetic Methods and Bioactivity Focused on Antidiabetic Potential. Chem. Rev. 2017, 117 (3), 1687-1764.

7. Ferrier, R. J.; Prasad, N., Unsaturated carbohydrates. Part IX. Synthesis of 2,3-dideoxy- $\alpha$-D-erythro-hex-2-enopyranosides from tri-O-acetyl-D-glucal. J. Chem. Soc. C 1969, o (4), 570-575.
8. Bhuma, N.; Lebedel, L.; Yamashita, H.; Shimizu, Y.; Abada, Z.; Ardá, A.; Désiré, J.; Michelet, B.; Martin-Mingot, A.; AbouHassan, A.; Takumi, M.; Marrot, J.; Jiménez-Barbero, J.; Nagaki, A.; Blériot, Y.; Thibaudeau, S., Insight into the Ferrier rearrangement by combining flash chemistry and superacids. Angew. Chem. Int. Ed. 2020, https://doi.org/10.1002/anie.202010175.

9. Lebedel, L.; Ardá, A.; Martin, A.; Désiré, J.; Mingot, A.; Aufiero, M.; Aiguabella Font, N.; Gilmour, R.; Jiménez-Barbero, J.; Blériot, Y.; Thibaudeau, S., Structural and Computational Analysis of 2-Halogeno-Glycosyl Cations in the Presence of a Superacid: An Expansive Platform. Angew. Chem. Int. Ed. 2019, 58 (39), 1375813762.

10. Mucha, E.; Marianski, M.; Xu, F.-F.; Thomas, D. A.; Meijer, G.; von Helden, G.; Seeberger, P. H.; Pagel, K., Unravelling the structure of glycosyl cations via cold-ion infrared spectroscopy. Nat. Commun. 2018, 9 (1), 4174.

11. Elferink, H.; Severijnen, M. E.; Martens, J.; Mensink, R. A.; Berden, G.; Oomens, J.; Rutjes, F.; Rijs, A. M.; Boltje, T. J., Direct Experimental Characterization of Glycosyl Cations by Infrared Ion Spectroscopy. J. Am. Chem. Soc. 2018, 140 (19), 6034-6038.

12. Marianski, M.; Mucha, E.; Greis, K.; Moon, S.; Pardo, A.; Kirschbaum, C.; Thomas, D. A.; Meijer, G.; von Helden, G.; Gilmore, K.; Seeberger, P. H.; Pagel, K., Remote Participation during Glycosylation Reactions of Galactose Building Blocks: Direct Evidence from Cryogenic Vibrational Spectroscopy. Angew. Chem. Int. Ed. 2020, 59 (15), 6166-6171.

13. Hansen, T.; Elferink, H.; van Hengst, J. M. A.; Houthuijs, K. J.; Remmerswaal, W. A.; Kromm, A.; Berden, G.; van der Vorm, S.; Rijs, A. M.; Overkleeft, H. S.; Filippov, D. V.; Rutjes, F.; van der Marel, G. A.; Martens, J.; Oomens, J.; Codee, J. D. C.; Boltje, T. J., Characterization of glycosyl dioxolenium ions and their role in glycosylation reactions. Nat. Commun. 2020, 11 (1), 2664.

14. Greis, K.; Mucha, E.; Lettow, M.; Thomas, D. A.; Kirschbaum, C.; Moon, S.; Pardo-Vargas, A.; von Helden, G.; Meijer, G.; Gilmore, K.; Seeberger, P. H.; Pagel, K., The Impact of Leaving Group Anomericity on the Structure of Glycosyl Cations of Protected Galactosides. ChemPhysChem 2020, 21 (17), 1905-1907.

15. Schöllkopf, W.; Gewinner, S.; Junkes, H.; Paarmann, A.; von Helden, G.; Bluem, H. P.; Todd, A. M. M., The new IR and THz FEL facility at the Fritz Haber Institute in Berlin. Proc. SPIE Int. Soc. Opt. Eng. 2015, 95121L.

16. Supady, A.; Blum, V.; Baldauf, C., First-Principles Molecular Structure Search with a Genetic Algorithm. J. Chem. Inf. Model. 2015, 55 (11), 2338-48.

17. Tkatchenko, A.; Scheffler, M., Accurate molecular van der Waals interactions from ground-state electron density and free-atom reference data. Phys. Rev. Lett. 2009, 102 (7), 073005.

18. Blum, V.; Gehrke, R.; Hanke, F.; Havu, P.; Havu, V.; Ren, X.; Reuter, K.; Scheffler, M., Ab initio molecular simulations with numeric atom-centered orbitals. Comput. Phys. Commun. 2009, 180 (11), 2175-2196.

19. Adamo, C.; Barone, V., Toward reliable density functional methods without adjustable parameters: The PBE0 model. J. Chem. Phys. 1999, 110 (13), 6158-6170.

20. Grimme, S.; Antony, J.; Ehrlich, S.; Krieg, H., A consistent and accurate $a b$ initio parametrization of density functional dispersion correction (DFT-D) for the 94 elements H-Pu. J. Chem. Phys. 2010, 132 (15), 154104.

21. Frisch, M. J. et al. Gaussian 16 Rev. A.03, Wallingford, CT, 2016.

22. Cremer, D.; Pople, J. A., General definition of ring puckering coordinates. J. Am. Chem. Soc. 1975, 97 (6), 1354-1358.

23. Hill, A. D.; Reilly, P. J., Puckering coordinates of monocyclic rings by triangular decomposition. J. Chem. Inf. Model. 2007, 47 (3), 1031-5. 